\title{
Long term protective level of hepatitis B antibody after revaccination in liver transplanted children: an open-label randomized control trial study
}

Palittiya Sintusek ${ }^{1}$, Yong Poovorawan², Supranee Buranapraditkun ${ }^{1}$, Piyaporn Wanawongsawad ${ }^{3}$, Ai-lada Intrarakamhang ${ }^{3}$

\footnotetext{
${ }^{1}$ Thai Pediatric Gastroenterology, Hepatology and Immunology Research Unit, Faculty of Medicine, King Chulalongkorn Memorial Hospital, Chulalongkorn University, The Thai Red Cross Society, Bangkok, Thailand

${ }^{2}$ Division of Gastroenterology, Department of Pediatrics, Faculty of Medicine, King Chulalongkorn Memorial Hospital, Chulalongkorn University, Bangkok, Thailand

${ }^{3}$ Center for Organ Transplantation, King Chulalongkorn Memorial Hospital, Chulalongkorn University, The Thai Red Cross Society, Bangkok, Thailand
}

Background: High prevalence of hepatitis B (HB)-antibody loss after liver transplantation (LT) was documented and reimmunization is merit. This study aims to evaluate the long-term protective level of HB surface antibody (anti-HBs) after HB revaccination in liver transplanted children.

Methods: Liver-transplanted children with previously immunization but anti-HBs after $\mathrm{LT} \leq 100 \mathrm{mlU} / \mathrm{mL}$ were recruited and randomized to reimmunization with standard-3-dose (SD) and double-3-dose (DD) HB vaccine intramuscularly at 0-1-6 months. Participants with anti-HBs $<100 \mathrm{mlU} / \mathrm{mL}$ after reimmunization was defined as antibody loss. Antibody loss rate was estimated using Kaplan-Meier method and the difference between antibody loss from SD and DD was compared using log-rank test. To assess the variable associated with antibody loss over time, multivariable Cox proportional hazard regression analysis was performed. Results: From 2016 to 2020, 68 children were recruited. The rate of anti-HBs $\geq 100 \mathrm{mlU} / \mathrm{mL}$ after complete vaccination was $87.1 \%$ and $80 \%$ in SD and DD. After the median follow-up period of 2.21 years $(1.34,2.96)$ from enrollment, geometric mean titer of anti-HBS was $199.32 \mathrm{mIU} / \mathrm{mL}(101.37-391.93)$ and $129.80 \mathrm{mlU} / \mathrm{mL}(60.59-278.08)$ in SD and $\mathrm{DD}(\mathrm{P}=0.419)$. There were $64.5 \%$ and $56.7 \%$ of participants with anti-HBs $>100 \mathrm{mlU} / \mathrm{mL}$ in SD and $\mathrm{DD}(\mathrm{P}=0.530)$. The median for antibody loss over time were 3.01 ( $95 \%$ confidence interval [Cl], 2.865-3.155) and $2.69(95 \% \mathrm{Cl}, 2.341-3.049)$ years for SD and DD. There was no significant antibody loss over time between both groups $(P=0.156)$. On the univariate analysis, low anti-HBs level at the enrollment was the only factor associated with increased risk of antibody loss over time (median anti-HBs of $2.2 \mathrm{mIU} / \mathrm{mL}$; hazard ratio, 3.403; 1.631-7.099; $\mathrm{P}<0.001)$.

Conclusions: SD and DD for HB reimmunization were highly effective to maintain the protective level of anti-HBs in liver-transplanted children after long-term follow-up. Moreover, early HB reimmunization should be scheduled for liver-transplanted children at the time that anti-HBs level is not too low.

Corresponding author: Palittiya Sintusek

E-mail: palittiya.s@chula.ac.th

(C) The Korean Society for Transplantation

This is an Open Access article distributed under the terms of the Creative Commons Attribution Non-Commercial License (http://creativecommons.org/licenses/by-nc/4.0/) which permits unrestricted non-commercial use, distribution, and reproduction in any medium, provided the original work is properly cited. 